\title{
VACUUM STRUCTURE OF 2+1-DIMENSIONAL GAUGE THEORIES
}

\author{
MANUEL ASOREY, FERNANDO FALCETO \\ JOSE LUIS LOPEZ AND GLORIA LUZON \\ Departamento de Física Teórica, Facultad de Ciencias, Universidad de Zaragoza \\ 50009 Zaragoza, Spain \\ E-mail: asorey@saturno.unizar.es
}

\begin{abstract}
We analyse some non-perturbative properties of the Yang-Mills vacuum in twodimensional spaces in the presence of Chern-Simons interactions. We show that the vacuum functional vanishes for some gauge field configurations. We have identified some of those nodal configurations which are characterized by the property of carrying a non-trivial magnetic charge. In abelian gauge theories this fact explains why magnetic monopoles are suppressed by ChernSimons interactions. In non-abelian theories it suggests a relevant role for nodal gauge field configurations in the confinement mechanism of Yang-Mills theories. In topological Chern-Simons theories nodal configurations belong to Atiyah-Bott strata with non-null codimension in the space of gauge field configurations. In the presence of external static quarks some nodes of the vacuum functional with non-trivial magnetic charge are removed and they are responsible for the increase of vacuum energy.
\end{abstract}

1. Introduction. The dual superconductor picture of the Quantum Chromodynamics vacuum ([20], [21], [24], [25]) assigns a leading role to magnetic monopoles in the quark confinement mechanism. The magnetic superconducting character of the quantum vacuum is induced by a condensation of magnetic monopoles. The chromo-electric flux is, then, expelled from the vacuum by the dual Meissner effect in a similar way as the magnetic field is expelled from a standard superconductor in the Meissner effect. The immersion of a quark-antiquark pair in such a magnetic superconducting vacuum generates a concentration of the chromo-electric flux lines along the string connecting the two particles, which induces an effective quark-antiquark potential growing linearly with the distance. Quark confinement is a consequence of such a linear interaction which only admits quark-antiquark bound states. In other words, the monopole condensation associates a finite tension to the string connecting the quark and the antiquark which does

1991 Mathematics Subject Classification: Primary 81T13; Secondary 81T40.

The paper is in final form and no version of it will be published elsewhere. 
not admit an infinite separation of the two particles.

Although, such a picture has not been confirmed as the real mechanism responsible for quark confinement in $\mathrm{QCD}_{3+1}$, a recent analysis carried out by Seiberg and Witten [30] suggests that it could be the real confinement mechanism in $N=2$ supersymmetric Yang-Mills theories.

The role of monopoles also seems to be crucial for quark confinement in $2+1$ dimensions. In compact lattice $\mathrm{QED}_{2+1}$ it has been shown that the logarithmic perturbative Coulomb potential becomes linear by means of Debye screening of electric charges in a monopole gas ([26], [27]) in a similar manner as vortices drive the Kosterlitz-Thouless phase transition in the XY model [10].

On the other hand, in 2+1-dimensional gauge theories there exists the possibility of having massive gluons while keeping gauge invariance. This is possible because in $2+1-$ dimensional space-time massless and massive vector-like particles have the same number of degrees of freedom. The generation of mass for gauge fields can be explicitly achieved in local terms thanks to the peculiar properties of the Chern-Simons term.

In such a case quarks are deconfined because no condensation of pseudoparticles can dramatically modify the exponential decay of gauge propagators. In fact, it has been shown that in compact $\mathrm{QED}_{2+1}$ the confinement of electric charges is traded by that of magnetic monopoles [1] (see also [16]), and the magnetic superconductivity picture of confinement is traded by a standard electric superconducting scenario. In this sense a topological mass perturbation realizes an electromagnetic duality transformation.

On the other hand it has been suggested by Feynman that in absence of nodes in the ground state of pure Yang-Mills theory in $2+1$ dimensions could be relevant for the confinement of quarks [18]. Such a property can be qualitatively derived from a generalization of the min-max principle. However, in the presence of the Chern-Simons interaction the min-max principle cannot be applied because of the complex character of the interaction. Moreover, it has been shown that all physical states in such a theory do have nodal points, i.e. the quantum functionals vanish for some classical configuration of the gauge fields.

If there is any relationship between the absence of confinement and the existence of nodes in the vacuum state of the theory with a Chern-Simons term it is possible to establish a connection between the configurations where the vacuum vanishes and permanent confinement.

In this paper we analyse the role of magnetic monopoles and nodes in the vacuum structure of topologically massive Yang-Mills theory. The case of abelian gauge theories is exactly solved and the role played by magnetic monopoles in the low energy regime illuminates the possible behaviour of non-abelian theories.

2. Canonical Quantization of Topologically Massive Yang-Mills Theory. Let $M_{3}$ be a 2+1-dimensional space-time. Any principal bundle $P\left(M_{3}, S U(N)\right)$ defined over $M_{3}$ with structure group $S U(N)$ is isomorphic to the trivial bundle $P=M_{3} \times S U(N)$. Therefore, in $2+1$ dimensions $S U(N)$ gauge fields $B$ can be considered as connections on the trivial bundle $P=M_{3} \times S U(N)$. The dynamics of topologically massive $S U(N)$ 
gauge fields on $M_{3}$ is governed by the action

$$
S_{\alpha}(B)=\frac{k}{4 \pi} \int_{M_{3}} \operatorname{Tr}\left(B \wedge d B+\frac{2}{3} B \wedge B \wedge B\right)+\frac{1}{2 \alpha^{2}}\|F(B)\|^{2} .
$$

Here || || denotes the norm associated to the scalar products of p-forms

$$
(\tau, \eta)=-2 \int_{M_{3}} \operatorname{tr} \tau \wedge * \eta
$$

$F(B)=d B+[B, B]$ is the curvature of the connection $B$ and $*$ is the Hodge operator associated to the (oriented) space-time metric $\left(M_{3}, g\right)$. Now, $S_{\alpha}(B)$ is not univocally defined for gauge fields $B$ of $P=M_{3} \times S U(N)$, because the value of the Chern-Simons term depends on the choice of the section which trivializes $P$ in expression (2.1). Indeed, $S_{\alpha}(B)$ is not invariant under large gauge transformations $\phi: M_{3} \rightarrow S U(N)$,

$$
S_{\alpha}\left(B^{\phi}\right)=S_{\alpha}(B)+2 \pi k \nu i,
$$

with non-trivial winding number

$$
\nu=\frac{1}{4 \pi^{2}} \int_{M_{3}} \operatorname{Tr} \phi^{-1} d \phi \wedge \phi^{-1} d \phi \wedge \phi^{-1} d \phi .
$$

Therefore, a consistent definition of the euclidean functional integral

$$
\int \delta B \mathrm{e}^{i S_{\alpha}(B)}
$$

is only possible for integer values of Chern-Simons charge $k$.

Let us consider a space-time of the form $M_{3}=\Sigma \times \mathbb{R}$, where $\Sigma$ is Riemann surface, endowed with a direct product metric. If we consider the temporal gauge $\left(B_{0}=0\right)$, the only degrees of freedom are the spatial components $A$ of the gauge fields $B=\left(A, B_{0}\right)$ and we can perform a canonical approach. The configuration space $\mathcal{A}_{\Sigma}$ is the space of connections $A$ defined over the Riemann surface $\Sigma$ with $S U(N)$ as structure group. The phase space is the cotangent bundle $T^{*} \mathcal{A}_{\Sigma}$, which can be parametrized by the connections $A \in \mathcal{A}_{\Sigma}$ and the associated canonical momenta $\Pi$. These momenta are constrained by Gauss law [8]

$$
d_{A}^{*}\left(\Pi+\frac{k}{4 \pi} * A\right)=\frac{k}{2 \pi} * F(A)
$$

induced by the motion equation of the temporal component $B_{0}$ of connection $B . d_{A}^{*}$ denotes the adjoint of the covariant derivative $d_{A}$ operator with respect to the scalar product defined on $\mathcal{A}_{\Sigma}$ by $(2.2)$, i.e. $d_{A}^{*}=-* d_{A} *$.

The Gauss law constraint (2.3) is also associated to the symmetry of the dynamics under the group $\mathcal{G}$ of base preserving automorphisms of the principal bundle $\Sigma \times S U(N)$. The final reduced phase space of the constrained system is the cotangent bundle $T \mathcal{M}$ of the space of gauge orbits $\mathcal{M}=\mathcal{A} / \mathcal{G}$, defined by the orbits of the action of $\mathcal{G}$ on $\mathcal{A}_{\Sigma}$ (from here on denoted $\mathcal{A}$, for simplicity).

The classical hamiltonian is given by

$$
H=(\pi, A)-L=\frac{\alpha^{2}}{2}\left\|\Pi+\frac{k}{4 \pi} * A\right\|^{2}+\frac{1}{2 \alpha^{2}}\|F(A)\|^{2} .
$$


In the Schrödinger representation canonical quantization gives the following prescription for the momentum operator

$$
\mathrm{III}=-i \frac{\delta}{\delta A} .
$$

The quantum hamiltonian $\mathbb{H}$ is then obtained by introducing such a prescription for the quantum momentum $\mathbb{I I}$ into the expression (2.4) of the classical hamiltonian $\left({ }^{1}\right)$. The quantum states are given by the complex functionals $\psi(A)$ on $\mathcal{A}_{\Sigma}$ (from here on denoted $\mathcal{A}$, for simplicity) which satisfy the quantum Gauss law condition

$$
-i d_{A}^{*} \frac{\delta}{\delta A} \psi(A)=\frac{k}{4 \pi} * d A \psi(A)
$$

This condition has a simple geometric interpretation in terms of the hermitean $U(1)$ connection $\widetilde{\alpha}_{k}$ defined over $\mathcal{A}$ by the one-form ([8], [2])

$$
\tilde{\alpha}_{k}(\tau)=\frac{k}{4 \pi}(* A, \tau)+\frac{k}{2 \pi}\left(d_{A} G_{A} * F(A), \tau\right) \quad \forall \tau \in T_{A} \mathcal{A}
$$

with $G_{A}=\left(d_{A}^{*} d_{A}\right)^{-1}$. Actually, the quantum Gauss law condition (2.6) can be written as

$$
d_{A}^{*} \nabla_{\tilde{\alpha}_{k}} \psi(A)=0
$$

with

$$
\nabla_{\tilde{\alpha}_{k}}=\frac{\delta}{\delta A}+i \widetilde{\alpha}_{k}
$$

which means that the quantum states are covariantly constant along the gauge fibres with respect to the connection $\widetilde{\alpha}_{k}$.

THEOREM 1. There exist non-trivial solutions of the quantum Gauss condition (2.8) if and only if $k$ is an integer.

Proof. The existence of solutions of the Gauss law requires the connection $\widetilde{\alpha}_{k}$ to be trivial along the orbits of the group of gauge transformations $\mathcal{G}([8],[3])$. The curvature form of $\widetilde{\alpha}_{k}$ is given by

$$
\widetilde{\Omega}_{k}(\widetilde{\tau}, \widetilde{\eta})=-\frac{k}{4 \pi}\left(\widetilde{\tau}^{h}, * \widetilde{\eta}^{h}\right)+\frac{k}{2 \pi}\left(G_{A} *\left[\widetilde{\tau}^{h}, \widetilde{\eta}^{h}\right], * F(A)\right),
$$

where $\tau^{h}$ is the component of $\tau$ which is orthogonal to the gauge fibre at $A$ with respect to the product defined by (2.2) i.e. $\widetilde{\tau}^{h}=P_{A} \widetilde{\tau}, P_{A}=\left(I-d_{A} G_{A} d_{A}^{*}\right)$ being the corresponding orthogonal projector. $\widetilde{\Omega}_{k}$ vanishes for vectors $\widetilde{\tau}, \widetilde{\eta} \in T_{A} \mathcal{A}$ tangent to the gauge fibres $\widetilde{\tau}=d_{A} \phi$. However, $\widetilde{\alpha}_{k}$ is trivial only if the holonomy group associated to any closed curve contained in a gauge orbit is trivial. This is only possible if the projection $\Omega_{k}$ of the curvature 2 -form $\widetilde{\Omega}_{k}$ to the space of gauge orbits $\mathcal{M}=\mathcal{A} / \mathcal{G}$,

$$
\Omega_{k}(\tau, \eta)=\widetilde{\Omega}_{k}\left(\widetilde{\tau}^{h}, \widetilde{\eta}^{h}\right)
$$

belongs (modulo a factor $2 \pi$ ) to an integer cohomology class of $\mathcal{M}$, i.e.

$$
\frac{1}{2 \pi}\left[\Omega_{k}\right] \in H^{2}(\mathcal{M}, \mathbb{Z}) .
$$

$\left({ }^{1}\right)$ There is not ordering problem in the kinetic term because all orderings give rise to the same quantum operator $\mathbb{H}$. 
In (2.10) $\widetilde{\tau}^{h}$ denotes the horizontal component of any tangent vector $\widetilde{\tau} \in T_{A} \mathcal{A}$ with projection $\tau \in T_{[A]} \mathcal{M}$.

Condition (2.11) is satisfied if and only if the Chern-Simons charge $k$ is an integer ([8], [7]).

In this way the quantization condition of $k$ also arises in the canonical formalism. Although, the above derivation of this consistency condition is very different of that given in the covariant functional integral formalism, both have a common origin: invariance under base preserving automorphisms of $\Sigma \times S U(N)$ with non-trivial winding number [8]. Because of the triviality of $\widetilde{\alpha}_{k}$ when $k$ is an integer, the action of the group of gauge transformations $\mathcal{G}$ can be globally lifted to an action on the line bundle $\mathcal{A} \times \mathbb{C}$. Gauss law implies the invariance of the quantum states under this action. Thus, the quantum states can be completely characterized by sections of the line bundle $\mathcal{E}_{k}(\mathcal{M}, \mathbb{C})$ defined by the gauge orbits $\mathcal{E}_{k}=\mathcal{A} \times \mathbb{C} / \mathcal{G}$ of such an action. This proves the following

Proposition 2. Solutions of Gauss law constraint (2.8) are in one-to-one correspondence with sections of the line bundle $\mathcal{E}_{k}(\mathcal{M}, \mathbb{C})$.

In the same way, the connection $\widetilde{\alpha}_{k}$ of $\mathcal{A} \times \mathbb{C}$ projects down to a connection $\alpha_{k}$ in $\mathcal{E}_{k}$ and the quantum hamiltonian can be expressed as an operator $([8],[2])$

$$
\mathbb{H}=\frac{\alpha^{2}}{2}\left\|\nabla_{\alpha_{k}}\right\|^{2}+\frac{1}{2 \alpha^{2}}\|F(A)\|^{2}+\frac{k^{2} \alpha^{2}}{8 \pi^{2}}\left(* F(A), G_{A} * F(A)\right)
$$

acting on the sections of $\mathcal{E}_{k}$. Therefore the quantum dynamics of the topologically massive Yang-Mills theory is very similar to that of the magnetic monopole on $S^{2}$ and the quantum Hall effect on a torus. The similarity with the quantum mechanical models suggests the existence of low energy physical effects related to the topological structure of the orbit space.

Definition 3. A nodal point $x \in M$ of a section $\xi$ of a line bundle $P(M, \mathbb{C})$ is a point of the base manifold where the section vanishes, i.e. $\xi(x)=0$.

THEOREM 4. Every physical state has nodal configurations on $\mathcal{M}$.

Pr o of. The Chern class of the line bundle $\mathcal{E}_{k}(\mathcal{M}, \mathbb{C})$ is non-trivial $c_{1}\left(\mathcal{E}_{k}\right)=\left[\Omega_{k}\right] / 2 \pi$. Then, the bundle $\mathcal{E}_{k}(\mathcal{M}, \mathbb{C})$ is non-trivial and it is easy to show that any section (i.e. any physical state) must vanish at some gauge field configurations [3].

Remark 5. Such a behaviour is in contrast with Feynman's claim on the absence of nodes in the ground state of pure Yang-Mills theory in $2+1$ dimensions [18] $\left.{ }^{2}\right)$. If there is any relationship between the absence of confinement and the existence of nodes in the vacuum state of the theory with a Chern-Simons term it is possible to establish a connection between the configurations where the vacuum vanishes and permanent confinement in the pure Yang-Mills theory.

In quantum mechanics the existence of nodes in the ground state is usually related to its degeneracy. In such a case, the position of nodal points is not relevant because

$\left({ }^{2}\right)$ The min-max argument does not apply here because of the presence of complex terms in the first term of the hamiltonian operator (2.12). 
they change from one state to another. However, in topologically massive gauge theories it has been claimed that the vacuum is not degenerated [4]. In such a case, the nodal points are also unique and the corresponding gauge configurations can play a role in the confinement mechanism.

The relevance of nodes was anticipated in [3], however, nodal configurations of the vacuum functional where unknown for a long time. In the present paper we will show the solution for this longstanding problem [6].

3. Abelian Theory. In the abelian case, $G=U(1)$, there is no topological reason for physical states to have nodes. If the space is compactified to become a $2 \mathrm{D}$ sphere $S^{2}$ the first and second homotopy groups of the orbit space vanish, $\pi_{1}(\mathcal{M})=\pi_{2}(\mathcal{M})=0$ $([9],[7])$ and, thus, any line bundle over $\mathcal{M}$ is trivial. Physical states are sections on a trivial bundle and, thus, they can be non-null for any gauge field configuration.

The space of orbits splits into several disconnected pieces $\left(\pi_{0}(\mathcal{M})=\mathbb{Z}\right)$ each one containing abelian gauge fields carrying the same magnetic charge. Since the magnetic charge is quantized by Dirac condition, the different connected components of $\mathcal{M}$

$$
\mathcal{M}=\bigcup_{n=-\infty}^{\infty} \mathcal{M}_{n}
$$

are parametrized by an integer number (the first Chern number)

$$
n=\frac{1}{2 \pi} \int_{\Sigma} F(A)=c_{1}(A)
$$

From a topological viewpoint all the connected components $\mathcal{M}_{n}$ of $\mathcal{M}$ are homeomorphic. Actually, they are diffeomophic to the component without magnetic charge $\mathcal{M}_{0}$.

If all the sections of the bundle $\mathcal{M} \times \mathbb{C}$ were physical states, the Hilbert space would be a sum $\mathcal{H}=\bigoplus_{n=-\infty}^{\infty} \mathcal{H}_{n}$ of Hilbert spaces, each one corresponding to different monopole backgrounds. The energies from each $n$-monopole sector would be shifted by $n^{2} / 2 \alpha^{2}$ by the effect of the potential term of the hamiltonian.

However this is not the case because Gauss law imposes a very restrictive condition on physical states.

THEOREM 6. Physical states vanish on the gauge orbits of connections with non-trivial first Chern class.

Proof. If we integrate both sides of the Gauss law (2.8)

$$
\int_{\Sigma} * d^{*} \frac{\delta}{\delta A} \psi(A)=\frac{i k}{4 \pi} \int_{\Sigma} F(A) \psi(A)
$$

the left-hand side vanishes by Stokes theorem, because under the integral we have a pure differential whereas the right-hand side reduces to $(k n / 2) \psi(A), n$ being the magnetic charge carried out by the gauge field $A$. Consequently, for gauge fields with non-trivial magnetic charge $(n \neq 0)$ the wave functional $\psi(A)$ must vanish. This means that when $k \neq 0$ only the sections over the $\mathcal{M}_{0}$ sector do correspond to physical states, i.e. $\mathcal{H}_{\text {phys }} \equiv$ $\mathcal{H}_{0}$. 
Actually, the theory is exactly solvable and the vacuum state reads

$$
\Psi_{0}(A)=\exp \left\{\frac{i k}{4 \pi}\left(d^{*} A, \Delta^{-1} * F(A)\right)-\frac{1}{2 \alpha^{2}}\left(* F(A), \Delta^{-1}\left(m^{2}+\Delta\right)^{\frac{1}{2}} * F(A)\right)\right\}
$$

for gauge fields without magnetic charge

$$
c_{1}(A)=\int_{\Sigma} F(A)=0
$$

and vanishes $\Psi_{0}(A)=0$ for magnetic monopole configurations $\left(c_{1}(A) \neq 0\right)$. The spectrum corresponds to a free massive photon with mass $m=k \alpha^{2} / 2 \pi([14],[15],[22])$. The basic property involved in the above argument is that constant gauge transformations do not transform abelian gauge fields which implies the vanishing of the left-hand side of the Gauss law whereas space constant temporal component of gauge fields $\left(A_{0}=\right.$ const) does couple to the other components by means of both, Yang-Mills and Chern-Simons, terms of the action, which leads to the equality of both sides of the Gauss law equation. In physical terms what happens is that the Chern-Simons term generates a transmutation of magnetic charge into electric charge which is reflected in the anomalous terms of Gauss law.

The same physical argument applies for higher genus $(g>0)$ compactifications $\Sigma$ of the physical space. In such a case the topology of the space of gauge orbits $\mathcal{M}$ becomes more sophisticate. It is given by

$$
\mathcal{M}=\mathbb{Z} \times \overbrace{S^{1} \times \ldots \times S^{1}}^{2 g \text { times }} \times \mathcal{H}
$$

where $\mathcal{H}$ is a real Hilbert space.

The above result can be easily derived from the splitting of the space of one-forms into its longitudinal, transverse and harmonic components,

$$
A=A_{0}+d \xi+* d \phi+\mathbf{a},
$$

where $A_{0}$ is any fixed $U(1)$-connection defined on the same line bundle than $A$, i.e. $A$ and $A_{0}$ have the same first Chern number

$$
c_{1}(A)=\frac{1}{2 \pi} \int_{\Sigma} F(A)=\frac{1}{2 \pi} \int_{\Sigma} F\left(A_{0}\right)=c_{1}\left(A_{0}\right) .
$$

The $\mathbb{Z}$ connected components of $\mathcal{M}, \mathcal{M}_{n}$ are parametrized by the magnetic monopole charge of the gauge fields, $c_{1}(A)$, and the $2 g-\left(S^{1}\right)$ components

$$
T^{g}=\overbrace{S^{1} \times \ldots \times S^{1}}^{2 g \text { times }}
$$

are generated by the harmonic forms a which must satisfy some periodic conditions in order to eliminate the overcounting of Gribov copies generated by large gauge transformations [9]. The factor $\mathcal{H}$ is associated to the transverse components $\phi$ of $A$. If $g \neq 0$ the curvature of the $U(1)$-connection $\Omega_{k}(2.10)$ belongs to an integer cohomology class $H^{2}(\mathcal{M}, \mathbb{Z})=H^{2}\left(T^{2 g}, \mathbb{Z}\right)=\mathbb{Z}^{2 g}$ if and only if $k \in \mathbb{Z}$. Consistency of the solutions under large gauge transformations requires, then, that for $g \neq 0$ the Chern-Simons charge $k$ be 
an integer $\left({ }^{3}\right)$. The physical states are in such a case sections of the bundle, $\mathcal{E}_{k}(\mathcal{M}, \mathbb{C})$. Since $\mathcal{E}_{k}(\mathcal{M}, \mathbb{C})$ is non-trivial because its first Chern class number $c_{1}\left(\mathcal{E}_{k}\right) \neq 0$, the quantization of the theory implies the existence of additional nodes also on the connected component of the orbit space, $\mathcal{M}_{0}$, defined by the abelian connections without magnetic charge $\left(c_{1}(A)=0\right)$. However in these cases the vacuum is degenerated and these configurations with vanishing amplitudes $\psi(A)=0$ are not physically relevant. They depend on the specific linear combination of vacuum states chosen. Any connection is in fact a node of a particular vacuum state. A basis of vacuum states is given by $\left\{\Psi_{0}^{n}, n=0,1, \ldots, k-1\right\}$, with

$$
\begin{aligned}
\Psi_{0}^{n}(A) & =\exp \left\{-k \pi \bar{u}(\operatorname{Im} \Omega)^{-1} u / 2+k \pi u(\operatorname{Im} \Omega)^{-1} u / 2\right\} \theta\left[\begin{array}{c}
n / k \\
0
\end{array}\right](k u ; k \Omega) \\
& \exp \left\{\frac{i k}{4 \pi}(\xi, \Delta \phi)-\frac{1}{2 \alpha^{2}}\left(\Delta \phi, \Delta^{-1}\left(m^{2}+\Delta\right)^{\frac{1}{2}} \Delta \phi\right)\right\}
\end{aligned}
$$

for gauge fields without magnetic charge, $c_{1}(a)=0([11],[12]) . \Psi_{0}^{n}$ vanish for any other gauge field configuration [5]. In expression $(3.4) u=\left(u^{i}\right)$ with $i=1, \ldots, g$, are the vectors of $\mathbb{C}^{g}$ which parametrize the harmonic forms $\mathbf{a}=i \pi u(\operatorname{Im} \Omega)^{-1} \omega$ in terms of a given basis of harmonic differentials $\omega=\left(\omega^{i}\right), \quad i=1, \ldots, g . \Omega$ is the matrix of periods associated to $\omega$ and a basis of $H_{1}(\Sigma)$. Besides those generic nodes, the states $\Psi_{0}^{n}$ also vanish at the zeros of the theta functions

$$
\theta\left[\begin{array}{c}
n / k \\
0
\end{array}\right](k u ; k \Omega) .
$$

From the above results, some of them anticipated in [4], we conclude that in $2+1-$ dimensional abelian gauge theories Chern-Simons interactions are absolutely incompatible with magnetic monopoles.

In the case $k=0$ the theory reduces to a pure Maxwell continuum (non-compact) theory. External charges are confined by a logarithmic potential. Monopoles are not confined and in fact they have a finite mass $M=1 / 2 \alpha^{2}$. When photons become massive by the effect of the Chern-Simons interaction electric charges are deconfined whereas magnetic monopoles decouple from the physical degrees of freedom, i.e. their mass becomes infinite and their correlators vanish. In this theory the dual superconductor picture, i.e. confinement/condensation of electric/magnetic charges, is explicitly realized and the addition of the Chern-Simons term makes the transition from one regime to another.

4. Non-abelian Theory. The same analysis holds in the non-abelian theory for gauge field configurations with non-zero total magnetic charge. However we will see that even in the sector of zero net charge there are some configurations where the states vanish.

Let us restrict ourselves, for simplicity, to the $S U(N)$ case, although our analysis can be easily generalized for arbitrary gauge groups. Gauge fields are defined on a trivial

$\left({ }^{3}\right)$ Strictly speaking Gauss law only imposes invariance under infinitesimal gauge transformations. If we do not impose invariance under large gauge transformations it is possible to obtain a consistent quantization for any value of $k$ ([28], [29]). This is equivalent to consider all the Gribov copies of the fields a as inequivalent field configurations. In such a case the relevant orbit space is $\mathbb{Z} \times H^{1}(\Sigma, \mathbb{R}) \times \mathcal{H}$. 
bundle $P=\Sigma \times S U(N)$. Reducible gauge fields $A$ can, actually, be defined on subbundles

$$
P_{r}\left(\Sigma, U\left(N_{1}\right) \times \ldots \times U\left(N_{r}\right)\right)
$$

of $P$ with structure group $U\left(N_{1}\right) \times \ldots \times U\left(N_{r}\right)$. They are decomposed into a sum $A=A_{1}+$ $A_{2}+\ldots+A_{r}$ of elementary gauge fields $A_{i}$ with values in $u\left(N_{i}\right)$. The gauge field elementary components $A_{i}$ of $A$ are defined on principal bundles $P_{i}\left(\Sigma, U\left(N_{i}\right)\right)$ whose first Chern classes $c_{1}\left(P_{i}\right)$ represent the magnetic charges of the different components of $A$. Since $A$ is a connection with gauge group $S U(N)$ the total magnetic charge $\sum_{i=1}^{r} c_{1}\left(P_{i}\right)=0$ vanishes.

Reducible gauge fields are invariant under the following group of gauge transformations

$$
\Phi_{t}=\left(\begin{array}{cccc}
\mathrm{e}^{i \mu_{1} t} I_{1} & 0 & \ldots & 0 \\
0 & \mathrm{e}^{i \mu_{2} t} I_{2} & \ldots & 0 \\
\ldots & \ldots & \ldots & \ldots \\
0 & 0 & \ldots & \mathrm{e}^{i \mu_{r} t} I_{r}
\end{array}\right)
$$

with $\mu_{i}=c_{1}\left(P_{i}\right) / N_{i}$ and where $I_{i}$ denotes the identity matrix of $U\left(N_{i}\right)$. Thus, the infinitesimal generator of the group $K_{t}$ of gauge transformations $\phi=\left.\dot{\Phi}_{t}\right|_{t=0}$ satisfies $d_{A} \phi=0$, and

$$
\int d^{2} x \operatorname{Tr} \phi d_{A}^{*} \frac{\delta}{\delta A} \psi(A)=0,
$$

for any functional $\psi(A)$. In particular, for physical states Gauss' law (2.8) implies that

$$
\int_{\Sigma} \operatorname{Tr} \phi d A \psi(A)
$$

must vanish. Now,

$$
\begin{aligned}
\int_{\Sigma} \operatorname{Tr} \phi d A & =\int_{\Sigma} \operatorname{Tr} \phi\left(d A+d_{A} A\right) \\
& =2 \int_{\Sigma} \operatorname{Tr} \phi F(A)=4 \pi \sum_{i=1}^{r} \frac{c_{1}\left(P_{i}\right)^{2}}{N_{i}},
\end{aligned}
$$

and, therefore, if one of the magnetic charges $c_{1}\left(P_{i}\right)$ of the components of $A$ is non-null every physical state must vanish at that gauge field configuration, i.e. $\psi(A)=0$.

However, reducible configurations do not exhaust all nodal configurations. The reason is that the topological arguments leading to the existence of nodes discussed at the beginning also apply to the orbit space of irreducible connections. Therefore, there should exist other genuine non-abelian configurations where physical states vanish. To find those configurations a more elaborate dynamical argument is required. In general, nodal configurations will not be the same for all physical states, only those with net magnetic charge satisfy this property. Non-abelian, irreducible nodes depend on the physical state we consider. In the following, we will analyse the nodes of the vacuum state.

To study the vacuum state we need to minimize expectation value the quantum hamiltonian $\mathbb{H}(2.12)$. For that it will be useful to introduce the chiral components of the connection. Having fixed a 2-dimensional metric $h$ our 2D space $\Sigma$ acquires a complex structure and this induces a chiral decomposition $A=A_{z} d z+A_{\bar{z}} d \bar{z}$ of the gauge field. 
The component $A_{\bar{z}}$ defines an holomorphic structure on the vector bundle $E\left(\Sigma, \mathbb{C}^{N}\right)$ associated to $P$. Conversely, once one fix an hermitean structure on $E$ any holomorphic structure on $E\left(\Sigma, \mathbb{C}^{N}\right)$ defines a unique unitary connection $A$ on $\mathrm{P}$. This correspondence induces an isomorphism between the space of gauge fields $\mathcal{A}$ and the space $\mathcal{A}_{\bar{z}}$ of holomorphic structures on $E\left(\Sigma, \mathbb{C}^{N}\right)$.

In terms of the chiral components of the gauge field the physical states that minimize the kinetic term of the hamiltonian (2.12) are of the form

$$
\psi(A)=\exp \left\{\frac{k i}{8 \pi} \int d z d \bar{z} \operatorname{Tr} A_{\bar{z}} A_{z}\right\} \xi\left(A_{\bar{z}}\right),
$$

$\xi\left(A_{\bar{z}}\right)$ being any holomorphic functional of $A_{\bar{z}}$. The restriction of the Gauss law to those states reads

$$
D_{\bar{z}} \frac{\delta}{\delta A_{\bar{z}}} \xi\left(A_{\bar{z}}\right)=\frac{k}{\pi} \partial_{z} A_{\bar{z}} \xi\left(A_{\bar{z}}\right),
$$

and it is an analogue to the Gauss law of pure Chern-Simons topological field theory. In fact, one can identify these states with those of the Chern-Simons theory in holomorphic quantization ([19], [4]).

In $\mathcal{A}_{\bar{z}}$ there is an action of a larger group of symmetries, the group of chiral or complex gauge transformations $\mathcal{G}_{\mathbb{C}}$. The action of $h \in \mathcal{G}_{\mathbb{C}}$ on $A_{\bar{z}}$ is given by

$$
{ }^{h} A_{\bar{z}}=h A_{\bar{z}} h^{-1}+i h \partial_{\bar{z}} h^{-1},
$$

and the isomorphism between $\mathcal{A}$ and $\mathcal{A}_{\bar{z}}$ induces an action of $\mathcal{G}_{\mathbb{C}}$ in $\mathcal{A}$ that extends the ordinary or unitary gauge transformations in $\mathcal{G}$.

The relevance of these transformations comes from the fact that integration of the Gauss law (4.5) determines how the states with minimal kinetic energy change under chiral gauge transformations of the gauge field. In [19] it is shown that the states are multiplied by a non-null factor depending on $A$ and the chiral gauge transformation $h \in \mathcal{G}_{\mathbb{C}}$. Now, we have shown that physical states must vanish for reducible gauge fields with magnetic monopole components, therefore the states with minimal kinetic energy also vanish along their complex orbits. Generically, the gauge fields in those orbits are non-reducible and thus we obtain this way a larger set of nodes for these states.

Atiyah and Bott [9] have studied in detail the action of the gauge group of chiral gauge transformations $\mathcal{G}_{\mathbb{C}}$ on $\mathcal{A}$ and they have shown that the chiral gauge orbits can be organized in strata of $\mathcal{A}$. The different strata are characterized by the Chern classes and ranks $\left(k_{i}, n_{i}\right)$ of the quotient (semi-stable) bundles $D_{i}=E_{i} / E_{i-1}$ of the canonical filtration of $E(\Sigma, \mathbb{C}), 0=E_{0} \subset E_{1} \subset \ldots \subset E_{r}=E$ of $E$ defined by the corresponding holomorphic structure. By construction, the slope $\mu_{i}=k_{i} / n_{i}$ of the different quotient bundles $D_{i}$ of any holomorphic structure is always a decreasing function, $\mu_{1}>\mu_{2}>$ $\ldots>\mu_{r}=0[9]$.

In every stratum there are abelian harmonic connections verifying the 2D Yang-Mills equations $d^{*} F(A)=0$. They are associated to holomorphic structures whose canonical filtration splits into a direct sum of quotient bundles, $E_{i}=D_{1} \oplus D_{2} \oplus \ldots \oplus D_{i}$. Therefore, the different Atiyah-Bott strata $\mathcal{A}_{\mu}$ can be parametrized by sequences of decreasing slopes $\mu=\left(\mu_{1}, \mu_{2}, \ldots, \mu_{r}\right)$ with $k_{1}+k_{2}+\ldots+k_{r}=0$, and every gauge field belongs to one of 
those strata, i.e.

$$
\mathcal{A}=\bigcup_{\mu} \mathcal{A}_{\mu}
$$

The (complex) codimension of the closure on an Atiyah-Bott strata $\mathcal{A}_{n}$ is

$$
\sum_{i>j}^{N}\left[n_{i} k_{j}-n_{j} k_{i}+n_{i} n_{j}(g-1)\right]
$$

where $g$ is the genus of the Riemann surface $\Sigma$.

One could argue that most of the fields belong to orbits of gauge fields without monopole components which define a dense set of $\mathcal{A}$. From a quantum point of view it means that they are the most relevant configurations for the dynamical behaviour of the theory. However, it turns out that they are the other orbits which are relevant for the discussion of the structure of vacuum states. In some sense, one can think of the space of gauge fields expanded by complex gauge transformations from configurations with nontrivial monopole components as a boundary of the space of all gauge field configurations. In this picture, the topological effects would arise as boundary conditions to be satisfied by the quantum states at those special configurations.

The biggest stratum, $\mathcal{A}_{0}$, is characterized by the fact that it contains the flat connections. It is an open dense submanifold of $\mathcal{A}$ and therefore it has null codimension. Most of the fields belong to this stratum. From a quantum point of view it means that they are the most relevant configurations for the dynamical behaviour of the theory. However, it turns out that they are the other orbits which are relevant for the discussion of the structure of vacuum states. In some sense, one can think of the space of gauge fields expanded by complex gauge transformations from configurations with non-trivial monopole components as a boundary of the space of all gauge field configurations. In this picture, the topological effects would arise as boundary conditions to be satisfied by the quantum states at those special configurations $\left({ }^{4}\right)$.

One simple physical way of characterizing higher order strata $\mathcal{A}_{\mu}$ with $\mu \neq 0$ is by the abelian magnetic monopoles and antimonopoles with globally vanishing magnetic charge that they contain. If $\Sigma=S^{2}$ and $N=2, \mathcal{A}_{0}$ is made of the connections which are gauge equivalent by a complex gauge transformation to the trivial flat connection $A=0$. Higher order strata are parametrized by a positive integer number $n>0$ and their gauge fields are gauge equivalent to a monopole-antimonopole doublet $E=L^{n} \oplus L^{-n}$ with magnetic charges $n$ and $-n$, respectively. For higher genus surfaces, the main stratum, $\mathcal{A}_{0}$, is made up of the gauge fields such that all subbundles of the associated holomorphic bundle $E$ have non-positive first Chern class. It is an open dense submanifold of $\mathcal{A}$. All flat connections belong to this stratum and the union of their complex gauge orbits is dense in $\mathcal{A}_{0}$. Then the physical states with minimal kinetic energy (4.4) are completely determined, like Chern-Simons states, by their values at flat connections [17].

$\overline{\left({ }^{4}\right)}$ The lower cohomology groups, $H^{0}(\mathcal{M}, \mathbb{Z})$ and $H^{1}(\mathcal{M}, \mathbb{Z})$ are determined by the topology of the main stratum $\mathcal{A}_{0}$, but the $\mathbb{Z}$ contribution of higher order strata to $H^{2}(\mathcal{M}, \mathbb{Z})$ is the origin of most of the topological effects of the model. 
In the case of the sphere $\Sigma=S^{2}$ since the chiral gauge orbit of the trivial connection $A=0$ expands the main stratum $\mathcal{A}_{0}$ of $\mathcal{A}$, there is a unique minimal state as in ChernSimons theory. Its wave functional $\psi_{0}$ is completely determined by its value at $A=0$ and it vanishes nowhere in $\mathcal{A}_{0}$. All other orbits have a gauge configuration decomposable into a sum of monopole/antimonopole gauge field components. Therefore, the physical states with minimal kinetic energy must vanish along all complex gauge orbits unless $\mathcal{A}_{0}\left({ }^{5}\right)$. This result is compatible with the fact that for any value of $\psi_{0}(0)$ the functional $\psi_{0}(A)$ converges to 0 as $A$ approaches an orbit of a field configuration with monopole components. A result that can be also obtained following the techniques of [19].

For higher genus Riemann surfaces the structure of the moduli space of flat connections is non-trivial and the states with minimal kinetic energy are not uniquely determined from the Gauss law (see [17] for the toroidal topology). There is a finite-dimensional space of physical states and they can have additional nodes for some particular flat connections. This nodes, however, are not physically relevant as they change from one state to another.

5. External Sources. In the presence of external sources the vacuum structure of the theory is modified. This can be easily understood in the abelian theory.

Let us consider the abelian theory with $Q$ electric static charges, $q_{i}$, sitting at the points $x_{i}$ of $\Sigma(i=1, \ldots, Q)$. The dynamics is governed by the new action

$$
S_{\alpha}^{Q}(B)=S_{\alpha}(B)+\sum_{i=1}^{Q} q_{i} B_{0}\left(x_{i}\right)
$$

Because of the static character of the charges, their current, $J_{\mu}=\delta_{\mu}^{0} \sum_{i=1}^{Q} q_{i} \delta\left(x-x_{i}\right)$, only couples to the temporal component $B_{0}$ of the electromagnetic field. Therefore, in the temporal gauge the value of the action, $S_{\alpha}^{Q}(B)$ does not depend on the charges. In particular the hamiltonian $\mathbb{H}$ is the same as in the pure gauge case (2.4). The only effect of the external charges is to modify the Gauss law which reads

$$
d^{*}\left(\Pi+\frac{k}{4 \pi} * A\right)=\frac{k}{2 \pi} * F(A)-\sum_{i=1}^{Q} q_{i} \delta\left(x-x_{i}\right) .
$$

The canonical quantization prescription (2.5) gives a new meaning to the quantum Gauss law. In terms of the $U(1)$-connection $\widetilde{\alpha}_{Q}$ defined by the one-form

$$
\begin{gathered}
\widetilde{\alpha}_{Q}(\tau)=\frac{k}{4 \pi}(* A, \tau)+\frac{k}{2 \pi}\left(\tau, d G\left[* F(A)-\frac{2 \pi}{k} \sum_{i=1}^{Q} q_{i} \delta\left(x-x_{i}\right)\right]\right) \\
\left.=\widetilde{\alpha}_{k}(\tau)-\left(\tau, d G \sum_{i=1}^{Q} q_{i} \delta\left(x-x_{i}\right)\right]\right),
\end{gathered}
$$

on the bundle $\mathcal{A} \times \mathbb{C}$, the quantum Gauss law condition (5.2) can be written as

$$
d_{A}^{*} \nabla_{\tilde{\alpha}_{Q}} \psi(A)=0
$$

$\left({ }^{5}\right)$ The determinant of chiral fermions also vanishes for the same gauge field configurations [23]. 
with

$$
\nabla_{\tilde{\alpha}_{Q}}=\frac{\delta}{\delta A}+i \widetilde{\alpha}_{Q}
$$

The condition (5.4) means that the quantum states are functionals of $\mathcal{A}$ which are covariantly constant along the gauge fibres with respect to the connection $\widetilde{\alpha}_{Q}$. The existence of solutions require the triviality of the connection $\widetilde{\alpha}_{Q}$ along such orbits. This condition is satisfied if and only if the curvature of the projection of $\widetilde{\alpha}_{Q}$ to $\mathcal{M}, \alpha_{Q}$, defines an integer 2 -form, i.e.

$$
\Omega_{Q}=d \alpha_{Q}=\Omega_{k} \in H^{2}(\mathcal{M}, \mathbb{Z}) .
$$

Since $\Omega_{Q}=\Omega_{k}$, according to Theorem 1 this implies that $k$ has to be an integer for Riemann surfaces $\Sigma$ with higher genus $g \geq 0$.

The integration of Gauss law leads to the following result.

TheOREM 7. Physical states vanish for connections whose Chern numbers $c_{1}(A)=$ $[1 / 2 \pi] \int_{\Sigma} * F(A)$ do not satisfy the quantization condition

$$
c_{1}(A)=\frac{2}{k} \sum_{i=1}^{Q} q_{i} .
$$

This implies that the quantum states have only fluctuations over connections which satisfy the constraint (5.6).

In particular the vacuum state is given by

$$
\begin{aligned}
& \Psi_{0}(A)= \exp \left\{\frac{i k}{4 \pi}\left(d^{*} A, \Delta^{-1}\left[* F(A)-\frac{4 \pi}{k} \sum_{i=1}^{Q} q_{i} \delta\left(x-x_{i}\right)\right]\right)\right\} \\
& \exp \left\{-\frac{1}{2 \alpha^{2}}\left(\left[* F(A)-\frac{m \alpha^{2}}{2}\left(m^{2}+\Delta\right)^{-1} \sum_{i=1}^{Q} q_{i} \delta\left(x-x_{i}\right)\right],\right.\right. \\
&\left.\left.\Delta^{-1}\left(m^{2}+\Delta\right)^{\frac{1}{2}}\left[* F(A)-\frac{m \alpha^{2}}{2}\left(m^{2}+\Delta\right)^{-1} \sum_{i=1}^{Q} q_{i} \delta\left(x-x_{i}\right)\right]\right)\right\}
\end{aligned}
$$

for gauge fields whose magnetic charge equals the total electric charge of the external electric sources,

$$
\int_{\Sigma} F(A)=\frac{4 \pi}{k} \sum_{i=1}^{Q} q_{i}
$$

and vanishes $\psi_{0}(A)=0$ for connections which do not satisfy such a condition. Notice that those configurations lead to singularities in the exponents of the vacuum functional. In the imaginary terms, because in such a case the harmonic component of $* F(A)-$ $\frac{4 \pi}{k} \sum_{i=1}^{Q} q_{i} \delta\left(x-x_{i}\right)$ is non-null and $\Delta^{-1}$ is not defined on such forms. However, the relevant singularities appear in the real part of the exponential. Indeed, the harmonic component of

$$
* F(A)-m \alpha^{2}\left(m^{2}+\Delta\right)^{-1} \sum_{i=1}^{Q} q_{i} \delta\left(x-x_{i}\right),
$$


does not vanish for such configurations, i.e.

$$
\int_{\Sigma} F(A) \neq \frac{2 \alpha^{2}}{m} \sum_{i=1}^{Q} q_{i} .
$$

Therefore it belongs to the kernel of the Laplace-Beltrami operator $\Delta$ and its inverse becomes singular for those terms.

The vacuum energy is also modified by the presence of the external sources. The energy shift is given by

$$
\Delta \mathcal{E}_{Q}=\frac{\alpha^{2}}{2} \sum_{i \neq j}^{Q} q_{i} q_{j}\left(\Delta^{2}+m^{2}\right)^{-1}\left(x_{i}, x_{j}\right) .
$$

In the case of two external charges, $q_{1}, q_{2}$, the energy shift (5.8) has an exponential decay

$$
\Delta \mathcal{E}\left(x_{1}, x_{2}\right) \approx q_{1} q_{2} \mathrm{e}^{-m d\left(x_{1}, x_{2}\right)} \quad \text { for } d\left(x_{1}, x_{2}\right) \gg 1
$$

for large distances $d\left(x_{1}, x_{2}\right)$ between the external sources, whereas at short distances it exhibits an ultraviolet logarithmic divergent behaviour

$$
\Delta \mathcal{E}\left(x_{1}, x_{2}\right) \approx-q_{1} q_{2} \log \left(\operatorname{md}\left(x_{1}, x_{2}\right)\right) \quad \text { for } d\left(x_{1}, x_{2}\right) \ll 1 .
$$

This confirms the non-confining character of the theory at large distances whereas at short distances the interaction between charges remains coulombian.

In the case of higher genus surfaces, $g>0$, the same analysis shows that the degeneracy of the vacuum is not removed by the the presence of the external charges and the effective interaction between them at large/short distances is genus independent. In particular, the vacuum states are given by

$$
\begin{gathered}
\Psi_{0}^{n}(A)=\exp \left\{-k \pi \bar{u}(\operatorname{Im} \Omega)^{-1} u / 2+k \pi u(\operatorname{Im} \Omega)^{-1} u / 2\right\} \theta\left[\begin{array}{c}
n / k \\
0
\end{array}\right](k u ; k \Omega) \\
\exp \left\{\frac{i k}{4 \pi}\left(\xi,\left[\Delta \phi-\frac{4 \pi}{k} \sum_{i=1}^{Q} q_{i} \delta\left(x-x_{i}\right)\right]\right)-\frac{1}{2 \alpha^{2}}\left(\left[\Delta \phi+m \alpha^{2} \sum_{i=1}^{Q} q_{i} \delta\left(x-x_{i}\right)\right],\right.\right. \\
\left.\left.\Delta^{-1}\left(m^{2}+\Delta\right)^{\frac{1}{2}}\left[\Delta \phi+m \alpha^{2} \sum_{i=1}^{Q} q_{i} \delta\left(x-x_{i}\right)\right]\right)\right\}
\end{gathered}
$$

( $n=0,1, \ldots, k-1)$, for gauge fields whose magnetic charge equals the total electric charge of the external electric charges. The vacuum states (5.9) vanish for any other gauge field configuration.

The only effect of the external charges appears in the form of the stationary states and in the corresponding energies, which are given by (5.8).

6. Conclusions. Some of the above results can be generalized for non-abelian theories. In such a case we have considered only the vacuum structure associated to the kinetic term of the hamiltonian (2.12) but the true vacuum state should minimize the whole hamiltonian, which also includes a potential term. To understand how this term 
could affect the vacuum structure we need a more elaborate argument. The crucial remark is that, as we have seen, the vacuum as any other physical state has to vanish at reducible gauge field configurations with monopole components, but among those configurations there are the absolute minima of the potential term when we restrict to the corresponding strata [9]. This can be understood from the following remarks. The flow defined by the gradient field of the potential term is tangent to the complex gauge orbits and such a flow has critical points at reducible configurations that are solutions of Yang-Mills equations. These critical gauge configurations can be found in any strata of $\mathcal{A}$, and it can be shown that the negative modes of the second variation of the potential at these critical points are orthogonal to their strata, which implies that they are local minima of the potential restricted to the those strata. The fact that they are global minima follows from the results of Atiyah and Bott [9].

Vanishing of the wave functional $\Psi_{0}(A)$ along the chiral gauge orbits containing solutions of Yang-Mills equation with magnetic monopole components is, then, necessary to minimize the expectation value of $\mathbb{H}$. It is not only required for the minimization of the kinetic term, but also for that of the Yang-Mills potential term, and it is a consequence of Ritz variational principle. Both terms of the hamiltonian, the kinetic and potential terms conspire to make the vacuum to vanish on the orbits of gauge field configurations with monopole components [6]. It is obvious that we cannot extend this argument to higher energy states.

This result explains why in the limit of infinite topological mass $\alpha^{2} \rightarrow \infty$ we recover the Chern-Simons states which by the same argument also vanish for the same configurations, and are completely determined by their values at flat gauge field configurations [19]. A similar result holds for an arbitrary gauge group $G$.

In summary, magnetic monopoles in topologically massive gauge theories are suppressed in any physical state by kinematical constraints, but the gauge field configurations on their complex gauge orbits are also suppressed in the vacuum state. They only give non-trivial contributions to excited states. Since the topologically massive gauge theories is not confining it is natural to speculate about the connection between the existence of nodes and the absence of confinement induced by the Chern-Simons interaction. The analysis of the effect of external static sources on the vacuum energy supports such a conjecture. If this connection exists an important role will be played by those configurations in the confinement mechanism for pure gauge theories. So far, most of the confinement scenarios gave a leading role to magnetic monopoles. From the above analysis it might be inferred that the gauge fields which are chiral gauge equivalent to those monopoles also play a relevant role. This opens a new possibility for understanding the mechanism of permanent confinement in 2+1-dimensional gauge theories.

Acknowledgements. M. A. thanks the organizers of Symposium for the warm hospitality extended to him during his stay at the Banach Center. J. L. L. was supported by a MEC fellowship (FPI program) and G. L. by a CONAI (DGA) fellowship. We also acknowledge CICyT and E. U. (Human Capital and Mobility Program) for partial financial support under grants AEN94-0218 and ERBCHRX-CT92-0035. 


\section{References}

[1] I. Affleck, J. Harvey, L. Palla, G. W. Semenoff, The Chern-Simons Term Versus the Monopole, Nuclear Phys. B 328 (1989), 575-584.

[2] M. Asorey, Spin and Statistics in Topologically Massive Theories, Phys. Lett. B 174 (1986), 199-202.

[3] M. Asorey, Topological Effects in Yang-Mills Theory in 2+1 Dimensions, in: Fields and Geometry, Ed. A. Jadczyk, World Scientific, Singapore, 1986, 31-47.

[4] M. Asorey, Topological Phases of Quantum Theories. Chern-Simons Theory, J. Geom. Phys. 11 (1993), 63-94.

[5] M. Asorey, S. Carlip, F. Falceto, Chern-Simons States and Topologically Massive Gauge Theories, Phys. Lett. B 312 (1993) 477-485.

[6] M. Asorey, F. Falceto, J. L. Lopez, G. Luzon, Nodes, Monopoles and Confinement in 2 + 1-Dimensional Gauge Theories, Phys. Lett. B 349 (1995), 125-130.

[7] M. Asorey, P. K. Mitter, Cohomology of the Yang-Mills Gauge Orbit Space and Dimensional Reduction, Ann. Inst. H. Poincaré Phys. Théor. 45 (1986), 61-78.

[8] M. Asorey, P. K. Mitter, Cohomology of the Gauge Orbit Space and 2+1-Dimensional Yang-Mills Theory with Chern-Simons Term, Phys. Lett. B 153 (1985), 147-152.

[9] M. Atiyah, R. Bott, The Yang-Mills Equations over Riemann Surfaces, Philos. Trans. Roy. Soc. London Ser. A 308 (1982), 523-615.

[10] V. L. Berezinskii, Destruction of Long-Range Order in One-Dimensional and TwoDimensional Systems Having a Continuous Symmetry Group. I: Classical Systems, Zh. Eksper. Teoret. Fiz. 59 (1970), 907-920 (in Russian); translated as Soviet Physics JETP 32 (1971), 493-500.

[11] M. Bos, V. P. Nair, U(1) Chern-Simons and $c=1$ Conformal Blocks, Phys. Lett. B 223 (1989), 61-66.

[12] M. Bos, V. P. Nair, Coherent State Quantization of Chern-Simons Theory, Internat. J. Modern Phys. A 5 (1990), 959-988.

[13] M. Crescimanno, S. A. Hotes, Monopoles, Modular Invariance and Chern-Simons Theory, Nuclear Phys. B 372 (1992), 683-700.

[14] S. Deser, R. Jackiw, S. Templeton, Three-Dimensional Massive Gauge Theories, Phys. Rev. Lett. 48 (1982), 975-978.

[15] S. Deser, R. Jackiw, S. Templeton, Topologically Massive Gauge Theories, Ann. Physics 140 (1982), 372-411.

[16] M. C. Diamantini, P. Sodano, C. A. Trugenberger, Topological Excitations In Compact Maxwell-Chern-Simons Theory, Phys. Rev. Lett. 71 (1993), 1969-1972.

[17] F. Falceto, K. Gawędzki, Chern-Simons States at Genus One, Comm. Math. Phys. 159 (1994), 549-579.

[18] R. Feynman, The Qualitative Behavior of Yang-Mills Theory in $(2+1)$-Dimensions, Nuclear Phys. B 188 (1981), 479-512.

[19] K. Gawędzki, A. Kupiainen, SU(2) Chern-Simons Theory at Genus Zero, Comm. Math. Phys. 135 (1991), 531-546.

[20] G. 't Hooft, Gauge Theories with Unified Weak, Electromagnetic and Strong Interactions, High Energy Physics, Ed. A. Zichichi, Ed. Compositori, Bologna, 1976.

[21] G. 't Hooft, Topology of the Gauge Condition and new Confinement Phases in nonAbelian Gauge Theories, Nuclear Phys. B 190 [FS3] (1981), 455-478. 
[22] R. Jackiw, Gauge Theories in Three Dimensions (= at High Temperature), in: Gauge Theories of the Eighties, Eds. E. Ratio, J. Lindfords, Lecture Notes in Physics 181, Springer, 1983, 157-219.

[23] A. Kupiainen, J. Mickelsson, What is the Effective Action in Two Dimensions?, Phys. Lett. B 185 (1987), 107-110.

[24] S. Mandelstam, Vortices and Quark Confinement in on-Abelian Gauge Theories, Phys. Rep. 23 (1976), 245-249.

[25] S. Mandelstam, General Introduction to Confinement, Phys. Rep. 67 (1980), 109-121.

[26] A. Polyakov, Compact Gauge Fields and the Infrared Catastrophe, Phys. Lett. B 59 (1975), 82-84.

[27] A. Polyakov, Quark Confinement and Topology of Gauge Groups, Nuclear Phys. 120 (1977), 429-458.

[28] A. P. Polychronakos, On the Quantization of the Coefficient of the Abelian ChernSimons Term, Phys. Lett. B 241 (1990), 37-40.

[29] A. P. Polychronakos, Abelian Chern-Simons Theories and Conformal Blocks, preprint UFIFT-89-9 (1989).

[30] N. Seiberg, E. Witten, Electro-Magnetic Duality, Monopole Condensation, and Confinement in $N=2$ Supersymmetric Yang-Mills Theory, Nuclear Phys. B 426 (1994), 19-52; Erratum: ibid. 430 (1994), 485-486. 